\title{
Efficient Updates for Continuous Skyline Computations ${ }^{\star}$
}

\author{
Yu-Ling Hsueh ${ }^{\dagger}$ Roger Zimmermann ${ }^{\ddagger}$ Wei-Shinn $\mathrm{Ku}^{\S}$ \\ ${ }^{\dagger}$ Dept. of Computer Science, University of Southern California, Los Angeles, CA 90089 \\ ${ }^{\ddagger}$ Computer Science Department, National University of Singapore, Singapore 117543 \\ ${ }^{\S}$ Dept. of Computer Science and Software Engineering, Auburn University, Auburn, AL 36849 \\ \{hsueh@usc.edu, rogerz@comp.nus.edu.sg, weishinn@auburn.edu\}
}

\begin{abstract}
We address the problem of maintaining continuous skyline queries efficiently over dynamic objects with $d$ dimensions. Skyline queries are an important new search capability for multi-dimensional databases. In contrast to most of the prior work, we focus on the unresolved issue of frequent data object updates. In this paper we propose the $E S C$ algorithm, an Efficient update approach for Skyline Computations, which creates a pre-computed second skyline set that facilitates an efficient and incremental skyline update strategy and results in a quicker response time. With the knowledge of the second skyline set, ESC enables (1) to efficiently find the substitute skyline points from the second skyline set only when removing or updating a skyline point (which we call a first skyline point) and (2) to delegate the most time-consuming skyline update computation to another independent procedure, which is executed after the complete updated query result is reported. We leverage the basic idea of the traditional $B B S$ skyline algorithm for our novel design of a two-threaded approach. The first skyline can be replenished quickly from a small set of second skylines - hence enabling a fast query response time - while de-coupling the computationally complex maintenance of the second skyline. Furthermore, we propose the Approximate Exclusive Data Region algorithm $(A E D R)$ to reduce the computational complexity of determining a candidate set for second skyline updates. In this paper, we evaluate the $E S C$ algorithm through rigorous simulations and compare it with existing techniques. We present experimental results to demonstrate the performance and utility of our novel approach.
\end{abstract}

\section{Introduction}

Skyline query computations are important for multi-criteria decision making applications and they have been studied intensively in the context of spatiotemporal databases. Skyline queries have been defined as retrieving a set of points, which are not dominated by any other points. An object $p$ dominates $p^{\prime}$, if $p$ has more favorable values than $p^{\prime}$ in all dimensions. Some of the prior work on

\footnotetext{
* This research has been funded in part by NSF grants EEC-9529152 (IMSC ERC), IIS-0534761, NUS AcRF grant WBS R-252-050-280-101/133 and equipment gifts from the Intel Corporation, Hewlett-Packard, Sun Microsystems and Raptor Networks Technology. We also acknowledge the support of the NUS Interactive and Digital Media Institute (IDMI).
} 


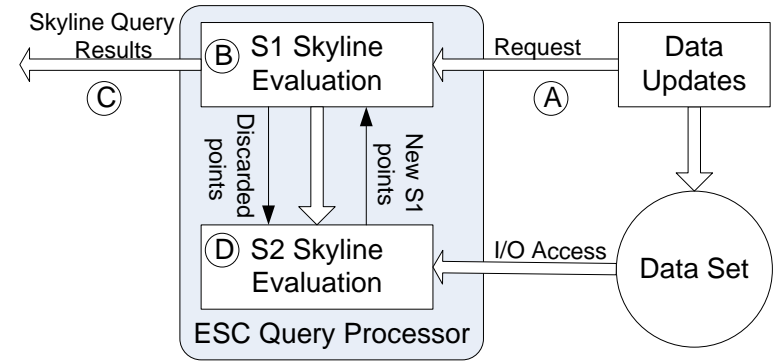

Fig. 1. ESC system framework

skyline queries assumed that data objects are static $[13,15]$. Other approaches assumed that the skyline computation involved only a partial of dynamic dimensions [4]. In this paper, we address Efficient Updates for Continuous Skyline Computations over dynamic objects (ESC for short), where objects with $d$ dynamic dimensions move in an unrestricted manner. Each dimension represents a spatial or non-spatial value. Towards an efficient continuous skyline computation the following challenges must be addressed: an effective incremental skyline query result update mechanism that is needed provides a fast response time of reporting the current query results, and an efficient strategy to reduce the search space dimensionality is required.

Existing work $[6,14,19]$ generally computes a number of data point subsets, each of which is exclusively dominated by one skyline point. Therefore, when a skyline point moves or is deleted, only its exclusively dominated subset must be scanned. The determination of such an exclusive data set is very computationally complex in higher dimensions and it incurs a serious burden for the system in a highly dynamic environment. Therefore, these systems are often unable to provide up-to-date query results with a quick response time. We propose the $E S C$ algorithm to efficiently manage the query results by delegating the time-consuming skyline update computations to another independent procedure, which is processed after the query processor reports the latest skyline query results. The key idea is to maintain a second skyline (or S2) set which is a skyline candidate set pre-computed when a traditional skyline (which we refer as the first skyline, $S 1$ ) point requests an update. With the knowledge of the second skyline set, the skyline query result can be updated within a limited search space and the expensive computations (e.g., searching for new second skylines to substitute a promoted second skyline point) can be decoupled from the first skyline update computations.

Figure 1 shows the framework of the ESC system. The query processor initially computes the first and second skyline points. Any updates (A) performed on the data set are also submitted to the query processor. First, Task (B) examines whether the update request (e.g., inserting or removing a data point) affects the first skyline set. If the request point becomes a new $S 1$ point, Task $\mathbf{B}$ inserts the new $S 1$ point into the current $S 1$ set and removes the current skyline points that are dominated by the new $S 1$ point. These discarded $S 1$ points (new 
$S 2$ points) are processed by Task (D) later to update the $S 2$ set. In case that an update request stems from a removed or moving $S 1$ point, some exclusive points are left un-dominated. The query processor searches for new substitute $S 1$ points only from the $S 2$ set. The query results (C) are immediately output as soon as Task (B) is completed. The processing time of the sequence of Tasks $(\mathbf{A})(\mathbf{B})(\mathbf{C})$ is the system response time to a skyline query update. Task (D) maintains the $S 2$ points when any $S 2$ point is inserted or removed. To enhance Task (D), which involves the expensive computation of determining exclusive data points where (D) searches for new or substitute $S 2$ points from the rest of the data set, we also propose an approximate exclusive data region computation with lower amortized cost than existing techniques [14,19]. The remainder of this paper is organized as follows. Section 2 describes the related work. Section 3 presents and details our continuous skyline query processing design. We extensively verify the performance of our technique in Section 4 and finally conclude with Section 5.

\section{Related Work}

Borzsonyi et al. [1] proposed the straightforward non-progressive Block-NestedLoop (BNL) and Divide-and-Conquer (DC) algorithms. The BNL approach recursively compares each data point with the current set of candidate skyline points, which might be dominated later. BNL does not require data indexing and sorting. The DC approach divides the search space and evaluates the skyline points from its sub-regions, respectively, followed by merge operations to evaluate the final skyline points. Both algorithms may incur many iterations and they are inadequate for on-line processing. In [17], Tan et al. presented two progressive processing algorithms: the bitmap approach and the index method. Bitmap encodes dimensional values of data points into bit strings to speed up the dominance comparisons. The index method classifies a set of $d$-dimensional points into $d$ lists, which are sorted in increasing order of the minimum coordinate. Index scans the lists synchronously from the first entry to the last one. With the pruning strategies, the search space is reduced. The nearest neighbor (NN) method [5] indexes the data set with an R-tree. NN utilizes nearest neighbor queries to find the skyline results. The approach repeats the queryand-divide procedure and inserts the new partitions that are not dominated by some skyline point into the to-do list. The algorithm terminates when the to-dolist is empty. In [13], a branch and bound skyline (BBS) algorithm traverses an R-tree to find the skyline points. Although BBS outperforms the NN approach, the performance can deteriorate due to many unnecessary dominance checks. Finally, many of the recent techniques aim at continuous skyline support for moving objects and data streams. Lin et al. [8] present $n$-of- $N$ skyline queries against the most recent $n$ of $N$ elements to support on-line computation against sliding windows over a rapid data stream. Morse et al. [11] propose a scalable LookOut algorithm for updating the continuous time-interval skyline efficiently. Sharifzadeh et al. [16] introduce the concept of Spatial Skyline Queries (SSQ). 
Given a set of data points $P$ and a set of query points $Q$, SSQ retrieves those points of $P$ which are not dominated by any other point in $P$ considering their derived spatial attributes to query points in $Q$. For moving query points, a continuous skyline query processing strategy is presented in [4] with a kinetic-based data structure. However, prompt query response is not considered in the design. A suite of novel skyline algorithms based on a Z-order curve [3] is proposed in [6]. Among the solutions, ZUpdate facilitates incremental skyline result maintenance by utilizing the properties of Z-order curve. Other related techniques can be found in the literature $[2,19,9,12,18]$. However, all the aforementioned studies differ from the main goal of this research - supporting frequent skyline data object updates efficiently while providing a quick response.

\section{ESC Algorithm}

\subsection{The Problem Definition of Continuous Skyline Queries}

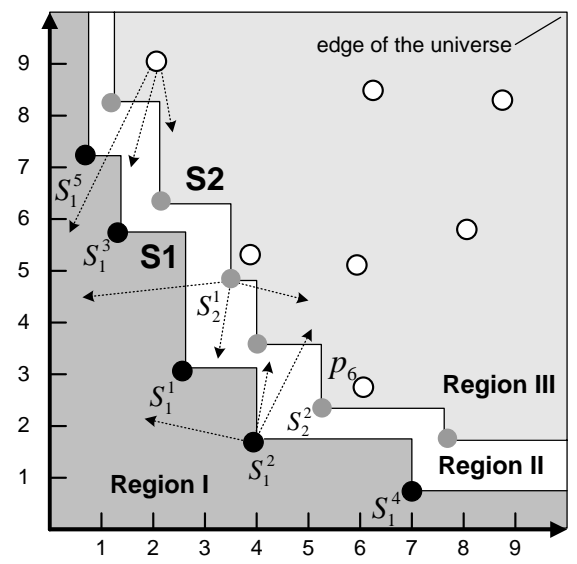

Fig. 2. $S 1$ and $S 2$ sets

The formal definition of skyline points in $d$-dimensional space is a distinct object set $P$, where any two objects $p=\left(x_{1}, \ldots, x_{d}\right)$ and $q=\left(y_{1}, \ldots, y_{d}\right)$ in the set satisfy the condition that if for any $k, x_{k}<y_{k}$, there exists at least one dimension of $m \leq d$ that satisfies $x_{m}>y_{m}$. We say $p$ dominates $q(p \vdash q$ for short), iff $x_{k}<y_{k}, \forall k(1 \leq k \leq d)$. The general setup of the problem consists of a set of dynamic query and data objects with $d$ dimensions. Moving objects can freely move in an unrestricted and unpredictable fashion, meaning that their parameters $x_{k}$ may arbitrarily change their values. The major challenging issue of a continuous skyline query is to avoid unnecessary dominance checking on irrelevant data points for skyline query result updates. After observing the $B B S$ algorithm [13], we deduced that when evaluating the skyline query result, a set of second skyline $(S 2)$ points can always be obtained with little extra work while 
retrieving the first skyline $(S 1)$ points. We refer to the traditional skyline query result as the first skyline, consisting of $S 1=\left\{s_{1}^{1}, \ldots, s_{1}^{m}\right\}$. The second skyline $S 2=\left\{s_{2}^{1}, \ldots, s_{2}^{k}\right\}$ is defined as follows:

Definition 1: A data point $p$ is a second skyline point iff $p \in(P-S 1)$ and $\nexists p^{\prime} \in(P-S 1-p), p^{\prime} \vdash p$. Informally, all $S 2$ points are dominated by $S 1$ and the rest of the data points $(P-S 1-S 2)$ are dominated by both $S 1$ and $S 2$.

When a $S 1$ point $s_{1}^{i}$ is removed or at least one value of its dimensions changes, the $S 2$ points are naturally considered as new $S 1$ point candidates to "substitute" $s_{1}^{i}$. The features of a $S 2$ set are as follows: (1) it is a pre-computed set that covers all the new $S 1$ candidate points, and (2) $S 2$ is a relatively small data set. Therefore, with the knowledge of $S 2$, the query processor can efficiently update the query result and provide a quicker response time to the query point. An example is shown in Figure 2. If a $S 1$ point $s_{1}^{2}$ moves to Region $I$, the search space for $E S C$ to update the query result only involves the $S 1$ set and the $S 2$ set. In this case, $s_{1}^{2}$ remains a $S 1$ point, but it dominates $s_{1}^{1}$. ESC needs to remove $s_{1}^{1}$ from the $S 1$ set and $s_{1}^{1}$ becomes a new $S 2$ point, since no existing $S 2$ point can dominate it. Due to the movement of $s_{1}^{2}, E S C$ searches for new $S 1$ points from the $S 2$ set. Since $s_{2}^{2}$ (an exclusive data point) is left un-dominated, $s_{2}^{2}$ becomes a new $S 1$ point and is removed from the $S 2$ set. The $E S C$ algorithm delegates the necessary $S 2$ maintenance (an independent procedure from $S 1$ updates) to the query processor after $S 1$ updates are completed. For example, new $S 2$ points must be retrieved to substitute $s_{2}^{2}$. To avoid scanning through the entire data points in Region $I I I$ for new $S 2$ points, we propose an approximate exclusive data region $(A E D R)$ computation in contrast to a traditional exclusive data region $(E D R)$ computation. Based on our observation and analysis, we provide the lemmas for incrementally updating the skyline query results in the following sections. Table 1 summarizes the symbols and functions we use throughout the following sections.

\begin{tabular}{|l|l|}
\hline Symbols & Descriptions \\
\hline$P$ & Number of data objects \\
\hline$d$ & Number of dimension \\
\hline$S 1$ & First skyline point set (traditional skyline query result set) \\
\hline$S 2$ & Second skyline point set \\
\hline DataRtree & Disk-based Rtree for indexing $P$ \\
\hline$S 1 R t r e e$ & Main-memory Rtree for indexing $S 1$ points \\
\hline$S 2 R t r e e$ & Main-memory Rtree for indexing $S 2$ points \\
\hline$E D R(p)$ & A set of data points in the exclusive data region \\
\hline$A E D R(p)$ & A set of data points in the approximate exclusive data region \\
\hline$W(p)$ & A set of skyline points in the dominance area of $p$ \\
\hline$p . D o m A r e a$ & The dominance area of $p$ \\
\hline
\end{tabular}

Table 1. Symbols and functions

\subsection{Second Skyline Computation}

The existing work $[14,19]$ performs the time-consuming exclusive data point computations for the skyline query result updates. In Figure 3, the gray areas 
represent the traditional EDRs that contain exclusive data points. An EDR is not usually pre-computed because of the complexity of the calculation. In contrast, since the $S 2$ points (new $S 1$ candidates) can be easily computed before any $S 1$ point issues an update, the query processor is able to satisfy a query request with the latest query result and with a quicker response time. To further reduce the search space of visiting $S 2$ points to update the skyline query result, we introduce and define a dominance set for each $S 1$ point $s_{1}^{i}$. A dominance set contains a group of $S 2$ points which are dominated by $s_{1}^{i}$ (denoted by $D\left(s_{1}^{i}\right)$ ) to substitute a removed or moving $s_{1}^{i}$ point when dominance relationship has changed. For example in Figure 3 the dominance set of $s_{1}^{2}$ includes $s_{2}^{2}$. If $s_{1}^{2}$ is removed, $E S C$ only checks the $S 2$ points in $D\left(s_{1}^{2}\right)$, instead of the entire $S 2$ points. In this example, $s_{2}^{2}$ becomes a new $S 1$ point, so it is removed from $S 2$. We formally define a dominance set and establish Lemma 1 which states that a dominance set must contain all the necessary $S 1$ candidate points as follows:

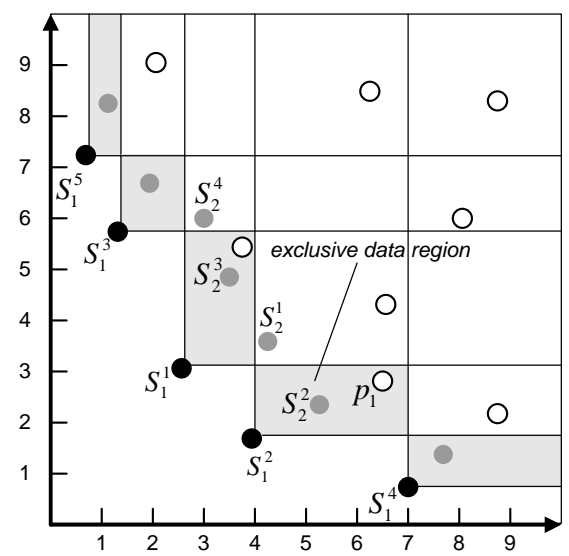

Fig. 3. Dominance set v.s. EDR set

Definition 2: (Dominance Set: $D\left(s_{1}^{i}\right)$ )

A dominance set of a skyline point $s_{1}^{i}$ (denoted by $D\left(s_{1}^{i}\right)=\left\{s_{2}^{r}, \ldots s_{2}^{v}\right\}$ ) is a $S 2$ subset where $\forall s_{2}^{w} \in D\left(s_{1}^{i}\right), s_{1}^{i} \vdash s_{2}^{w}$, and $0 \leq\left(s_{2}^{w}\right.$.mindist $-s_{1}^{i}$.mindist $) \leq$ $\left(s_{2}^{w}\right.$.mindist $-s_{1}^{t}$. mindist $), \forall s_{1}^{t} \in\left(S 1-s_{1}^{i}\right)$. Each $D\left(s_{1}^{i}\right)$ is exclusive from any other dominance set; therefore, $S 2=D(S 1)$, where $D(S 1)=D\left(s_{1}^{1}\right)+\ldots+D\left(s_{1}^{m}\right)$ and $m$ is the size of $S 1$.

Lemma 1: Given a $D\left(s_{1}^{i}\right)$. Let $A$ be the skyline points extracted from $\operatorname{EDR}\left(s_{1}^{i}\right) . D\left(s_{1}^{i}\right)$ must contain $A$ ( $A$ is a subset of $\left.\mathrm{D}\left(s_{1}^{i}\right)\right)$.

Proof: (By contradiction) Let $p \in A$ be a point not included in $D\left(s_{1}^{i}\right)$. This is a contradiction, since $p$ is only dominated by $s_{1}^{i}$. Therefore, it must be in $D\left(s_{1}^{i}\right)$. Therefore, $D\left(s_{1}^{i}\right)$ must contain all points in $A$.

In Figure $3, D\left(s_{1}^{2}\right)=\left\{s_{2}^{1}, s_{2}^{2}\right\}$ contains two $S 2$ points in the set which is a superset of $A=\left\{s_{2}^{2}\right\}$. One can observe that some non-exclusive $S 2$ points 
(e.g., $s_{2}^{1}$ and $s_{2}^{4}$ ) can be assigned to different dominance sets. Intuitively, the $S 1$ point with the minimal mindist to the query point (which has the largest dominance area) may contain the most $S 2$ points. Thus, it might produce a load imbalance problem because the query processor needs to perform many dominance checks when a skyline point with a short mindist moves. To ensure that each dominance set has evenly distributed $S 2$ points, the $E S C$ algorithm inserts a non-exclusive $S 2$ point $s_{2}^{w}$ into $D\left(s_{1}^{j}\right)$, where $s_{1}^{j}$ has the minimal value of $\left(s_{2}^{w}\right.$. minsit $-s_{1}^{j}$.mindist $)$ among all other $S 1$ points. In our algorithm, we utilize the $B B S$ approach to initially compute the skyline query results. Along with the query evaluation, $S 2$ points and the dominance set of each $S 1$ point are computed during the execution of the modified $B B S$ dominance-checking procedure which runs a window query to determine a set of candidate skyline points. Let $e$ be the next discarded entry during the process of the dominance-checking procedure ( $e$ is dominated by some $S 1$ point). Therefore, the algorithm proceeds to insert $e$ into a dominance set and examine whether $e$ is a $S 2$ point. Given is a heap $H=\left\{s_{1}^{i} \ldots s_{1}^{k}\right\}$ that is the set of the existing skyline points whose entries intersect with $e$. Since $B B S$ always visits entries in the ascending order of their mindist, we have $\forall s \in H, s$.mindist < e.mindist. With the sorting of $H$ by the mindist in descending order, $\exists s_{1}^{j} \in H, s_{1}^{j} \vdash e$ and the value of (e.minsit $-s_{1}^{j}$.mindist) $>0$ is minimal among all other $S 1$ points. Next, Lemma 2 is provided to prove the correctness of the $S 2$ extraction.

Lemma 2: Given a point $p$ which is dominated by $S 1^{\prime}=\left\{s_{1}^{i} \ldots s_{1}^{j}\right\}$, where $S 1^{\prime} \subset S 1$. If $\forall s_{2}^{t} \in D\left(S 1^{\prime}\right), s_{2}^{t} \nvdash p, p$ must be a $S 2$ point.

Proof: Since $p$ is not dominated by $\left(S 1-S 1^{\prime}\right), p$ can never be dominated by any $S 2$ point in $D\left(S 1-S 1^{\prime}\right)$ either, by transitivity. Therefore, if $p$ is not dominated by any $S 2$ point in $D\left(S 1^{\prime}\right), p$ is guaranteed to be a final $S 2$ point.

The pseudo code is shown in Algorithm 1, where the additional conditions (Lines 10-16 and 19-27) are inserted into the dominance-checking code for retrieving $S 2$ points and determining the dominance sets. Line 4 sorts the heap in descending order of the mindist such that the skyline points with larger mindist are examined first. Line 12 obtains the dominating skyline point $e_{r}$ for $p$ which is inserted into $D\left(e_{r}\right)$ later. Based on Lemma 2, Lines 13-15 check whether $p$ is a $S 2$ point. Lines 20-23 ensure that each $S 2$ is a data point. If $e$ is an intermediate node, $B B S$ is performed to retrieve local skyline points from the entry. Lines 23 and 25 insert the final $S 2$ points $O^{\prime}$ into $S 2$ and updates the $S 2$ set by deleting those $S 2$ points that are dominated by $O^{\prime}$. To find such a set, the algorithm performs S2Rtree.W(O $\left(O^{\prime}\right)$, which is a window query that finds the $S 2$ points in the dominance areas of $O^{\prime}$.

\subsection{Description of the ESC Algorithm}

The main procedures of the ESC algorithm include S1Evaluation for the $S 1$ updates and S2Evaluation for the $S 2$ set maintenance. ESC delegates most of expensive computations that are irrelevant to $S 1$ query results to S2Evaluation. 


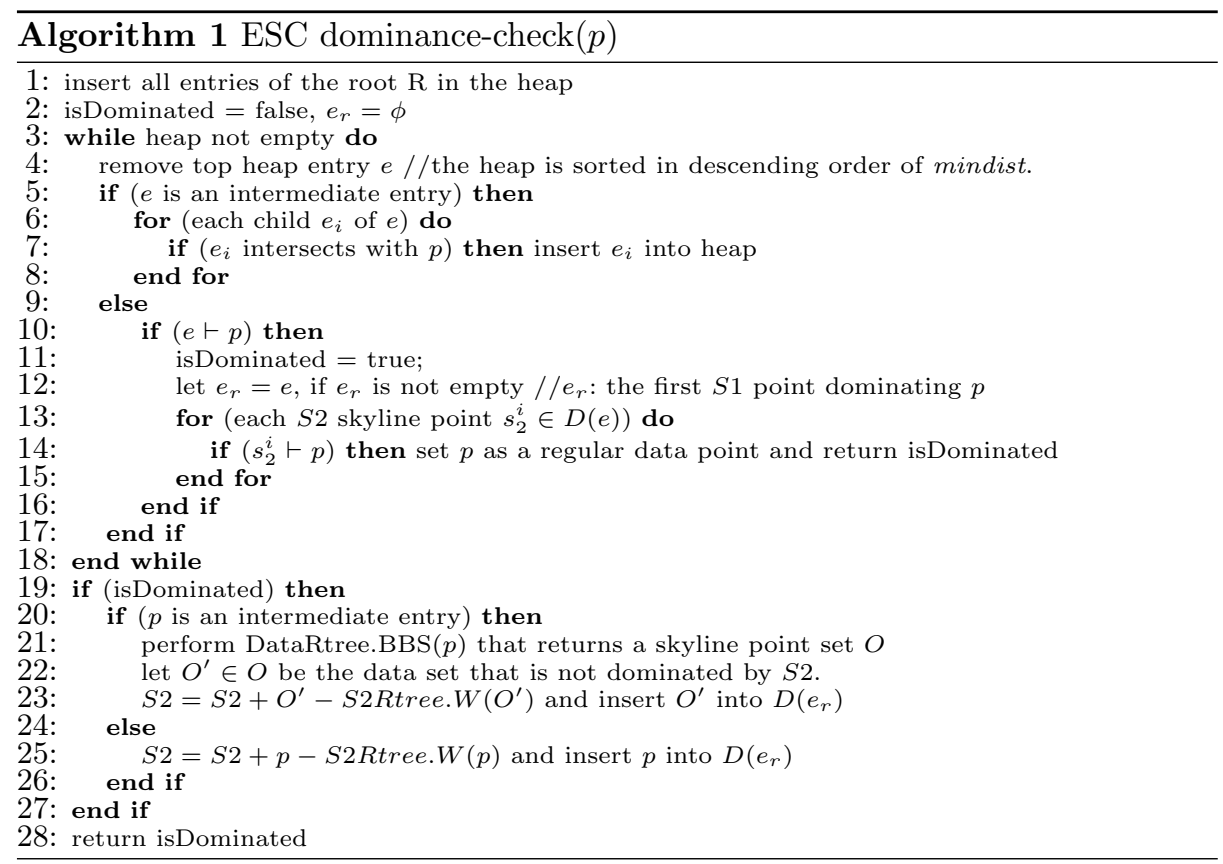

To improve the performance of S2Evaluation, we introduce the concept of an approximate exclusive data region $(A E D R)$ that helps to reduce the amortized cost of the $S 2$ updates. When $d=2$, the traditional $E D R$ is a regular rectangle. However, a $E D R$ has an irregular shape in higher dimensions. For example, in Figure $4(\mathrm{a}), s_{2}^{i}$ is a skyline point to delete. The $E D R$ is a irregular rectangle after deleting the overlapping area with the dominance area of $s_{2}^{k}$ and $s_{2}^{v}$. Based on this observation, we can obtain a regular shaped $E D R$ only when we consider the skyline points which have a value $x^{i}$ larger than that of $s_{2}^{i}$ in only one dimension. Because these points are completely "outside" of the $E D R$, they can trim the entire areas that represent the upper dimensional value $x^{i}$.

Definition 3: $(A E D R)$

Let $s_{2}^{i}=\left(x^{1}, x^{2}, \ldots, x^{d}\right)$, and $s_{2}^{j}=\left(y^{1}, y^{2}, \ldots, y^{d}\right)$. AEDR $\left(s_{2}^{i}\right)=s_{2}^{i}$.DomArea $\left(s_{2}^{i}\right.$.DomArea $\cap s_{2}^{j}$.DomArea $), \forall s_{2}^{j} \in\left(S 2-s_{2}^{i}\right)$, there exists exactly one $x^{k}<y^{k}$, $1 \leq k \leq d$.

For example, in Figure 4(b), $s_{2}^{i}$ is the skyline to delete and the solid rectangle box is an $A E D R$, which is a regular shape resulting from trimming the overlapping dominance areas of $s_{2}^{i}$ and $s_{2}^{j}$. ESC utilizes the $A E D R$ to search for the new $S 2$ points by traversing the R-tree. Each $M B R$ e extracted from the heap is checked whether it intersects with the $A E D R$. If true, ESC checks whether $e$ is dominated by the existing $S 2$ points.

When a $S 1$ point $p$ is newly inserted into the system or when it moves, $E S C$ needs to re-group a new dominance set for $p$. A simple solution is to check every $S 2$ point which currently belongs to a dominance set of some $S 1$ point 


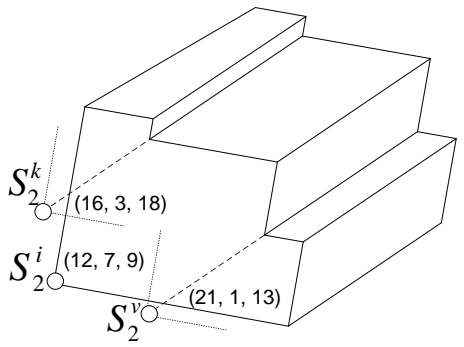

(a) 3-d EDR example

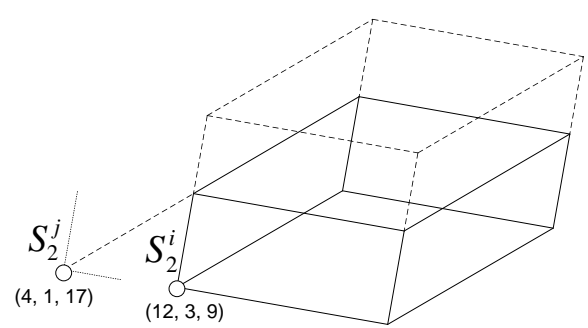

(b) AEDR example

Fig. 4. Traditional $E D R$ v.s. $A E D R$

and migrate the $S 2$ point to the dominance set of $p$ if necessary. Instead, we provide FindDomSet, (the pseudo code is presented in Algorithm 2) applying the following Lemma that presents a heuristic to avoid checking the entire $S 2$ set.

Lemma 3: Given a new $S 1$ point $s_{1}^{k}$, re-group the points in $D\left(s_{1}^{i}\right)$, only where $\forall s_{1}^{i} \in\left(S 1-s_{1}^{k}\right), s_{1}^{i}$.mindist $\leq s_{1}^{k}$.mindist.

Proof: Proof by definition. Let $s_{1}^{w}$ be a $S 1$ point that has the value of $\left(s_{1}^{w}\right.$.mindist $>s_{1}^{k} \cdot$ mindist $) . \forall p \in D\left(s_{1}^{w}\right)$, the value of (p.mindist $-s_{1}^{w} \cdot$ mindist) must be smaller than the value of ( .mindist $-s_{1}^{k}$.mindist). $p$ must remain in the same dominance set of $s_{1}^{w}$. Therefore, it is not necessary to re-group these points in $D\left(s_{1}^{w}\right)$.

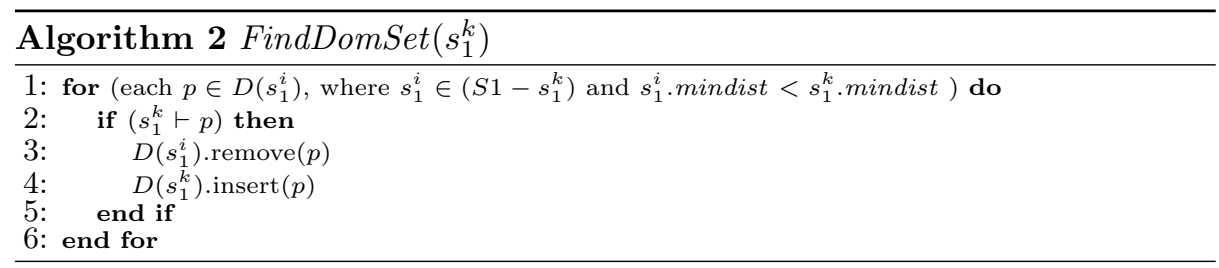

The ESC algorithm is implemented in an event-driven fashion to handle the skyline query updates. The main procedures include S1Evaluation (Algorithm 3) and S2Evaluation (Algorithm 4). When the query processor receives a request ( $S 1, S 2$, or regular data point), it first performs S1Evaluation to examine whether the request affects the $S 1$ set (the query result) and outputs the updated $S 1$ points if the set has been modified. Then S2Evaluation processes the rest of non-S1-related computations. In the S1Evaluation procedure, Line 6 performs the S1Rtree.dominace-descending function where the dominance checks access the S1Rtree in the descending order of the mindist of the entries. We use the same principle of the ESC dominance-check algorithm (discussed in Section 3.2) to find the dominating $S 1$ point $s_{1}^{k}$ (Line 7) for a request point $p$. If $p$ becomes a new $S 2$ point evaluated by S2Evaluation, $p$ is inserted into $D\left(s_{1}^{k}\right)$. 
Lines 9-10 update the $S 1$ set if $p$ is a new $S 1$ point and delete the $I$ set, which is an existing $S 1$ set dominated by $p . I$ is obtained by executing a window query S1Rtree. $W(p)$, using the dominance area of $p$ as the range on the S1Rtree. Line 11 inserts the new $S 1$ point $p$ into $\widetilde{S 1}$ and S1Evaluation will later pass this set to S2Evaluation where FindDomSet $(\widetilde{S 1})$ is performed to find a $S 2$ set for $D(p)$. Since all the points in $I$ become new $S 2$ points (inserted into $\widetilde{S 2}$ in Line 12), the $S 2$ set is updated later in S2Evaluation by adding the $\widetilde{S 2}$ set. Lines $15-24$ basically check all the $S 2$ points $\in D\left(p^{\prime}\right)$ whether they are still dominated by $p$ after $p$ moves or is removed from the system. In Line 18, since o (new $S 1$ point after $p$ moves) can never dominate any $S 1$ point, $o$ is added to the $S 1$ set directly. This is because $o$ is an exclusive data point, and therefore $o$ must not dominate any existing $S 1$ points.

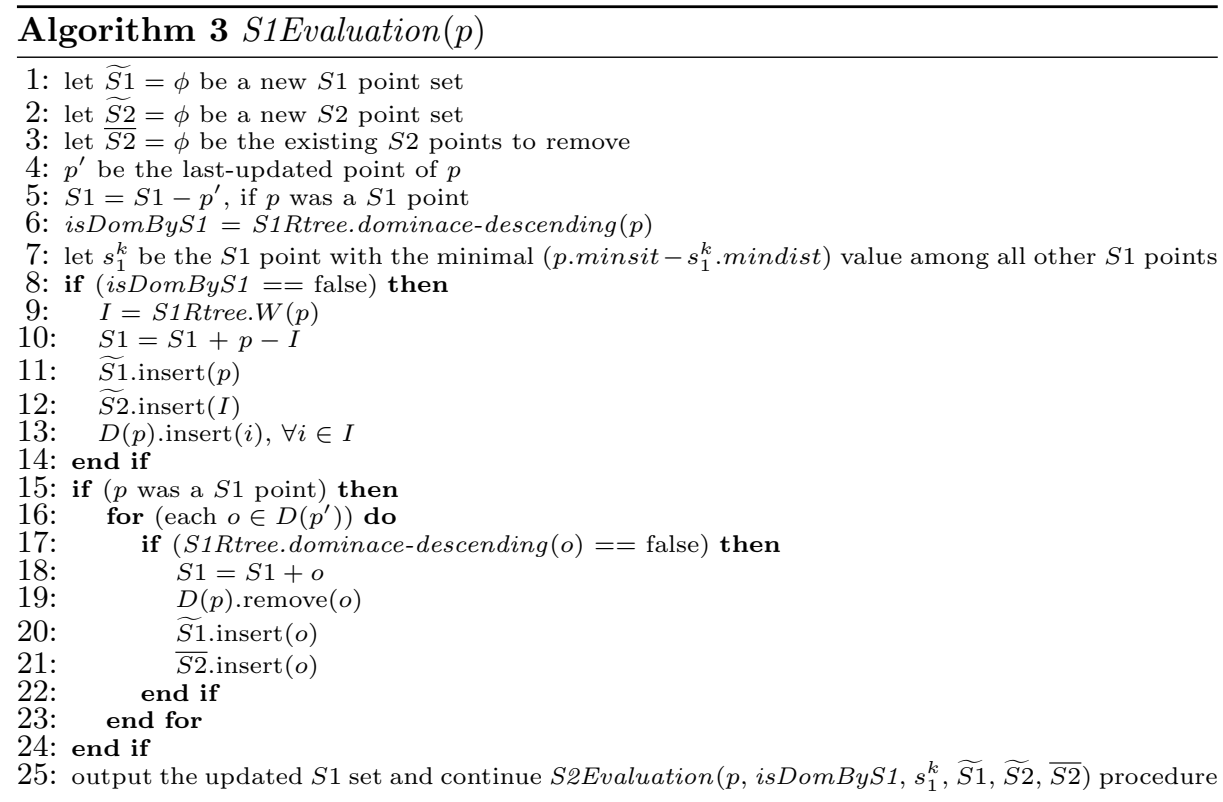

S2Evaluation is a more expensive procedure than S12Evaluation, because it involves $A E D R$ computations to find a set of new $S 2$ points to substitute a moving or removed $S 2$ point. Lines $6-7$ are processed if $p$ is a new $S 2$ point. The insertion of $p$ may dominate some existing $S 2$ points; therefore, Line 6 finds the dominated $S 2$ points (S2Rtree. $W(p)$ ) and removes them from the $S 2$ set. Similarly, in Line 10, since each point in $\widetilde{S 2}$ was originally a $S 1$ point, the $D(\widetilde{S 2})$ set is directly removed from the $S 2$ set without performing a window query to look for the dominated points. The deletion of the $S 2$ point set $\overline{S 2}$ is executed in Lines $11-12$ and $A$ contains the substitute $S 2$ points, after $\overline{S 2}$ is removed from the $S 2$ set. Finally, FindDomSet is performed to find a group of $S 2$ points for each point in $\widetilde{S 1}$. 


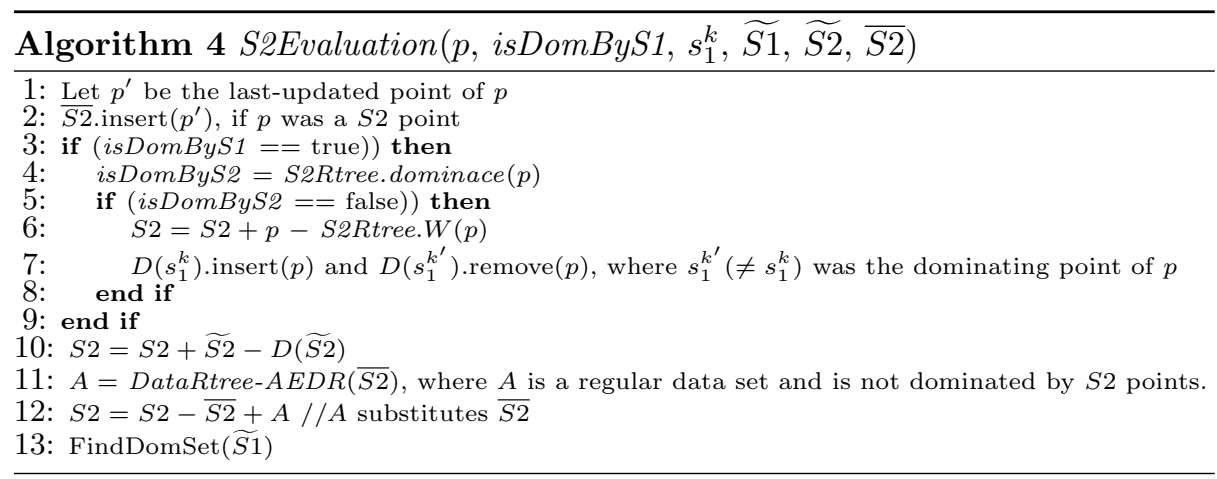

\section{Experimental Evaluation}

We evaluated the performance of the $E S C$ algorithm by comparing it with the well-known $B B S$ approach [14] and the DeltaSky algorithm [19]. For the $E D R$ computations in BBS, we adopt the $A B B S$ (Adaptive Branch-and-Bound Search) [19] to avoid complex irregular-shaped EDR computations. $A B B S$ basically traverses the R-tree and determines whether an intermediate $M B R e_{i}$ intersects with the dominance area of a skyline to delete. If this is true, it further checks whether any existing skyline dominates $e_{i}$. All of these algorithms utilize R-trees as the underlying structure for indexing the data and skyline points. We use the Spatial Index Library [7] for the R-tree index. A page size of 4 Kbytes is deployed, resulting in node capacities between $94(d=5)$ and 204 $(d=2) . S 1$ and $S 2$ sets are indexed by a main-memory R-tree to improve the performance of the dominance checks. Our data sets are generated on a terrain service space of $[0,1000]$ with the random walk mobility model [10]. Each object moves with a constant velocity until an expiration time. The velocity is then replaced by a new velocity with a new expiration time. We generated from 100,000 to 1,000,000 normal distributed data points with a dimension in the range of 2 to 5 . The object update ratio is set in a range from $1 \%$ to $10 \%$. Experiments are conducted with a Pentium $3.20 \mathrm{GHz}$ CPU and 1 GByte of memory. The query results are evaluated in an event-driven approach. Therefore, the query processor calls different procedures based on each specific event type. The main measurement in the following simulations is the response CPU time (from receiving a data update request to the $S 1$ update completion time or the evaluation time of S1Evaluation) and the overall CPU time (the evaluation time of S1Evaluation plus S2Evaluation). For $A B B S$ and DeltaSky the overall CPU time also represents the response time. Our experiments use several metrics to compare these algorithms. Table 2 summarizes the default parameter settings in the following simulations.

\begin{tabular}{|l|l|l|}
\hline Parameter & Default & Range \\
\hline$P$ & 100,000 & $100,000,500,000,1,000,000$ \\
\hline$d$ & 5 & $2,3,4,5$ \\
\hline$f_{\text {update }}$ & $10 \%$ & $1 \%, 5 \%, 10 \%$ \\
\hline
\end{tabular}

Table 2. Simulation parameters 


\subsection{Update Ratio}

First, we evaluated the impact of the update ratio. Figures 5(a) and (b) show the response time and overall CPU time as a function of update ratio, respectively, and Figure 5(c) illustrates the I/O cost for the three methods. We fix the data cardinality at 100,000 and dimensionality at 5. The ESC approach achieves a better performance than $A B B S$ and DeltaSky for all update rates. The degradation of DeltaSky is caused by the expensive Maximum Coverage computations scanning over the projection lists and the increase of skyline point size which incurs bigger projection lists. ESC also outperforms both methods in terms of the overall CPU time, since the amortized cost of the AEDR computations and exclusive data evaluation is lower than the other two methods.

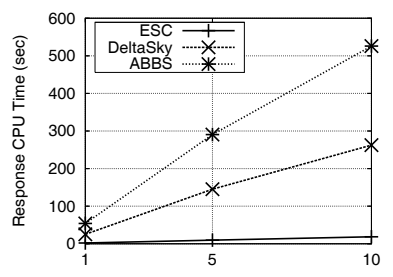

(a) Response CPU time

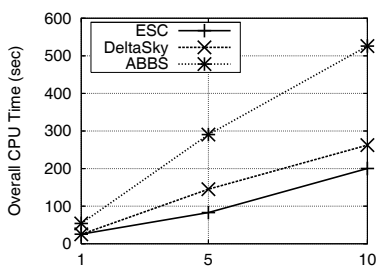

(b) Overall CPU time

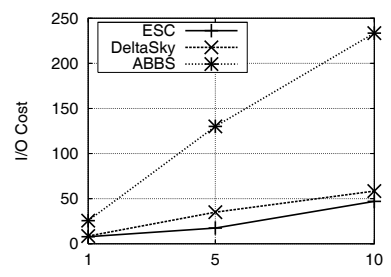

(c) $\mathrm{I} / \mathrm{O}$ cost

Fig. 5. Performance v.s. Update Ratio $(P=100 \mathrm{k}, d=5)$

\subsection{Dimensionality}

Next we report on the impact of the dimensionality on the performance of all three methods. Figures 6(a)(b)(c) show the CPU overheads and I/O cost v.s. the dimensionality ranging from $d=2$ to 5 , respectively. When $d$ increases, the performance of all methods is degraded because the exclusive data point computations are complex and R-trees fail to filter out irrelevant data entries in higher dimensions. From all the figures, we can see that $E S C$ outperforms $A B B S$ and DeltaSky in terms of the CPU time and I/O cost.

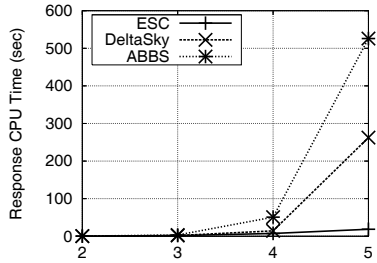

(a) Response CPU time

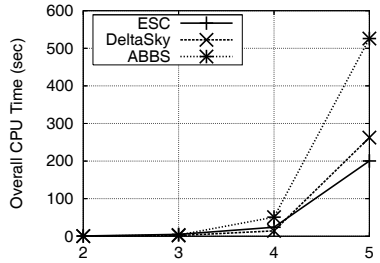

(b) Overall CPU time

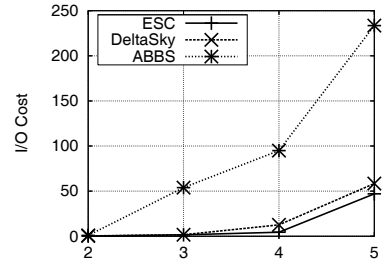

(c) $\mathrm{I} / \mathrm{O}$ cost

Fig. 6. Performance v.s. Dimensionality $\left(P=100 \mathrm{k}, f_{\text {update }}=10 \%\right)$ 


\subsection{Cardinality}

Figures 7(a)(b) show the response and overall CPU time as a function of the number of data points, respectively, and Figure 7 (c) illustrates the corresponding $\mathrm{I} / \mathrm{O}$ cost. Overall, the CPU overheads increase as a function of the number of data points. ESC achieves a significant reduction in terms of the response CPU time compared to $A B B S$ and DeltaSky. ESC takes advantage of the pre-computed $S 2$ points retrieved by the latest S2Evaluation procedure and quickly locates relevant new $S 1$ candidates for substituting a removed or moving $S 1$ point. As we can see from the experimental results, the adoption of $A E D R$ helps $E S C$ to achieve better overall CPU performance and competitive I/O cost with DeltaSky.

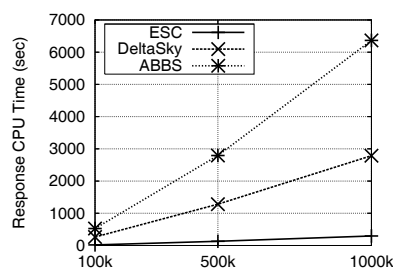

(a) Response CPU time

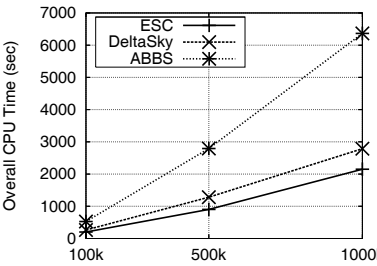

(b) Overall CPU time

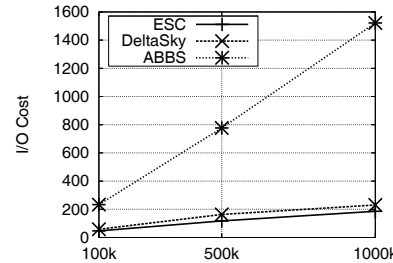

(c) I/O cost

Fig. 7. Performance v.s. Cardinality $\left(d=5, f_{\text {update }}=10 \%\right)$

\section{Conclusions}

In this paper, we propose an incremental skyline update approach. Our ESC algorithm achieves a faster response time and overall CPU performance. With the adoption of the pre-computed $S 2$ sets, $E S C$ can efficiently update the skyline query results and delegate the most complex computations to a separate procedure that executes after the updates of the query results are completed. An approximate exclusive data region $(A E D R)$ is proposed and our experiments confirm the feasibility of $A E D R$ which has a low amortized cost of the exclusive data evaluation in high dimensional and dynamic data environments. The S1Evaluation procedure first examines all the incoming data requests and updates the $S 1$ result if necessary and the S2Evaluation procedure integrates our lemmas and heuristics to achieve a low CPU overhead and reduced I/O cost.

\section{References}

1. S. Börzsönyi, D. Kossmann, and K. Stocker. The Skyline Operator. In Proceedings of the 17th International Conference on Data Engineering (ICDE), Heidelberg, Germany, pages 421-430, 2001.

2. C. Y. Chan, P.-K. Eng, and K.-L. Tan. Stratified computation of skylines with partially-ordered domains. In Proceedings of the ACM SIGMOD International Conference on Management of Data, Baltimore, Maryland, USA, pages 203-214, 2005. 
3. V. Gaede and O. Günther. Multidimensional Access Methods. ACM Comput. Surv., 30(2):170-231, 1998.

4. Z. Huang, H. Lu, B. C. Ooi, and A. K. H. Tung. Continuous Skyline Queries for Moving Objects. IEEE Trans. Knowl. Data Eng., 18(12):1645-1658, 2006.

5. D. Kossmann, F. Ramsak, and S. Rost. Shooting Stars in the Sky: An Online Algorithm for Skyline Queries. In Proceedings of 28th International Conference on Very Large Data Bases (VLDB), Hong Kong, China, pages 275-286, 2002.

6. K. C. K. Lee, B. Zheng, H. Li, and W.-C. Lee. Approaching the Skyline in Z Order. In Proceedings of the 33rd International Conference on Very Large Data Bases (VLDB), University of Vienna, Austria, pages 279-290, 2007.

7. S. I. Library. http://www.research.att.com/ marioh/spatialindex/index.html.

8. X. Lin, Y. Yuan, W. Wang, and H. Lu. Stabbing the Sky: Efficient Skyline Computation over Sliding Windows. In Proceedings of the 21st International Conference on Data Engineering (ICDE), Tokyo, Japan, pages 502-513, 2005.

9. X. Lin, Y. Yuan, Q. Zhang, and Y. Zhang. Selecting Stars: The k Most Representative Skyline Operator. In Proceedings of the 23rd International Conference on Data Engineering (ICDE), Istanbul, Turkey, pages 86-95, 2007.

10. A. B. McDonald. A mobility-based framework for adaptive dynamic cluster-based hybrid routing in wireless ad-hoc networks. Ph.D. Dissertation proposal, University of Pittsburgh, 1999., 1999.

11. M. D. Morse, J. M. Patel, and W. I. Grosky. Efficient Continuous Skyline Computation. In Proceedings of the 22nd International Conference on Data Engineering (ICDE), Atlanta, GA, USA, page 108, 2006.

12. M. D. Morse, J. M. Patel, and H. V. Jagadish. Efficient Skyline Computation over Low-Cardinality Domains. In Proceedings of the 33rd International Conference on Very Large Data Bases (VLDB), University of Vienna, Austria, pages 267-278, 2007.

13. D. Papadias, Y. Tao, G. Fu, and B. Seeger. An Optimal and Progressive Algorithm for Skyline Queries. In Proceedings of the 2003 ACM SIGMOD international conference on Management of data, pages 467-478, New York, NY, USA, 2003.

14. D. Papadias, Y. Tao, G. Fu, and B. Seeger. Progressive Skyline Computation in Database Systems. ACM Trans. Database Syst., 30(1):41-82, 2005.

15. J. Pei, W. Jin, M. Ester, and Y. Tao. Catching the Best Views of Skyline: A Semantic Approach Based on Decisive Subspaces. In Proceedings of the 31st International Conference on Very Large Data Bases (VLDB), Trondheim, Norway, pages 253-264, 2005.

16. M. Sharifzadeh and C. Shahabi. The spatial skyline queries. In Proceedings of the 32nd International Conference on Very Large Data Bases (VLDB), Seoul, Korea, pages 751-762, 2006.

17. K.-L. Tan, P.-K. Eng, and B. C. Ooi. Efficient Progressive Skyline Computation. In Proceedings of the 27th International Conference on Very Large Data Bases ( $V L D B$ ), pages 301-310, San Francisco, CA, USA, 2001. Morgan Kaufmann Publishers Inc.

18. L. Tian, L. Wang, P. Zou, Y. Jia, and A. Li. Continuous Monitoring of Skyline Query over Highly Dynamic Moving Objects. In Sixth ACM International Workshop on Data Engineering for Wireless and Mobile Access (MobiDE), Beijing, China, pages 59-66, 2007.

19. P. Wu, D. Agrawal, Ö. Egecioglu, and A. E. Abbadi. Deltasky: Optimal Maintenance of Skyline Deletions without Exclusive Dominance Region Generation. In Proceedings of the 23rd International Conference on Data Engineering (ICDE), The Marmara Hotel, Istanbul, Turkey, pages 486-495, 2007. 